\title{
A Visualization Toolkit For Transportation Simulation Systems
}

\author{
Banafsheh Hajinasab $^{1} \quad$ Paul Davidsson $^{1} \quad$ Jonas Löwgren $^{2} \quad$ Jan A. Persson $^{1}$ \\ ${ }^{1}$ Department of Computer Science, Malmö University \\ ${ }^{2}$ MEDEA Collaborative Media Initiative, Malmö University \\ \{Banafsheh.hajinasab, Paul.davidsson, Jonas.lowgren, Jan.a.persson\}@mah.se
}

\begin{abstract}
In this work, we investigate how visualization techniques could address the challenges of transportation simulation data analysis in order to facilitate the decision-making process for transportation simulation users. For this purpose, we have applied the visualization methods in a real implemented agent-based transportation simulator called TAPAS as a case. We have analyzed the visualization related requirements of users using a user-centric approach and an interactive visualization toolkit has been designed and developed based on the identified requirements. This paper presents a description of the visualization toolkit which will be used to investigate how the actors in a transport chain are expected to act when different types of governmental control policies are applied, such as, fuel taxes, road tolls, vehicle taxes and requirements on vehicles.
\end{abstract}

\section{Author Keywords transportation simulation visualization; transport planning.}

\section{ACM Classification Keywords}

H.5.2. Information interfaces and presentation (e.g., HCI): User Interfaces; D.2.2. Software Engineering: Design Tools and Techniques.

\section{INTRODUCTION}

There are many national and international models which predict future effects of the decisions in freight transport network [1]. These systems have a potential to assist in planning process where the decision makers wish to be aware of the consequences of a specific decision on the whole system. There are some factors which determine the degree of success in simulation systems. One important factor is the "Ease of use" of the system by potential users [1]. Typically, a simulation system generates a large amount of data which makes it a challenging task to analyze and present the data for different types of stakeholders in freight transport. Since individuals acquire more information through their sense of vision than through all the other senses [2]; Information visualization, as a way of presenting different data types in an understandable form, has the potential to support the analysis of transportation simulation data.

Copyright is held by the author/owner(s).

NordiCHI'12, Oct 14-17 2012, Copenhagen, Denmark.

ACM 978-1-4503-1482-4/12/10.

\section{RELATED WORK}

Although the use of visualization in transportation research is increasing, the majority of previous studies revolve around visualization of the transportation infrastructures (e.g. new road or bridge) to enhance the public awareness regarding upcoming projects which makes it easier to reach a consensus on the high-level decisions [5]. Another approach to visualization of transportation simulation is visualization of the simulation process (e.g. the appearing of loads and truck movements). The focus of this approach is on the visualization of how the simulation system works rather than visualization of the output results of the simulation [3]. In this approach, visualization is timedependent as opposed to our approach which is visualization of output data of simulation scenarios. For this purpose, we explore a multi-agent based simulation model for transportation and production called TAPAS [4].

\section{USER REQUIREMENTS ANALYSIS}

The first step seeks to understand the problems users experience regarding readability and usability of TAPAS output without visualization, and how a visualization tool could help them. To achieve this, we have conducted interviews with TAPAS users who are mostly experts in the field of transportation. Based on the results from interviews, the following user requirements are identified: Req1. Illustrating input and output of simulation; users prefer to see which input generates a specific output. Req2. Showing different scenarios concurrently; the users would like to be able to see several scenarios at the same time to be able to compare various scenarios and their related output. Req3. Showing simulation information based on links and routes; where link is a directed connection between two nodes and can have exactly one mode of transportation, and route is a set of links from a starting node to end node.

\section{DESIGN DECISIONS AND FUNCTIONALITIES OF VISUALIZATION TOOLKIT}

We have designed several mock-ups of decision decisions. These mock-ups are presented to the users in an iterative manner in order to support users to imagine the real system and facilitate the process of designing functionalities of the visualization toolkit. The proposed User Interface of the TAPAS visualization tool has two Tab Views; Link based view and Route based view. Relevant information of each category is displayed in their related tab. During discussions with users and presenting the mock-ups, we 


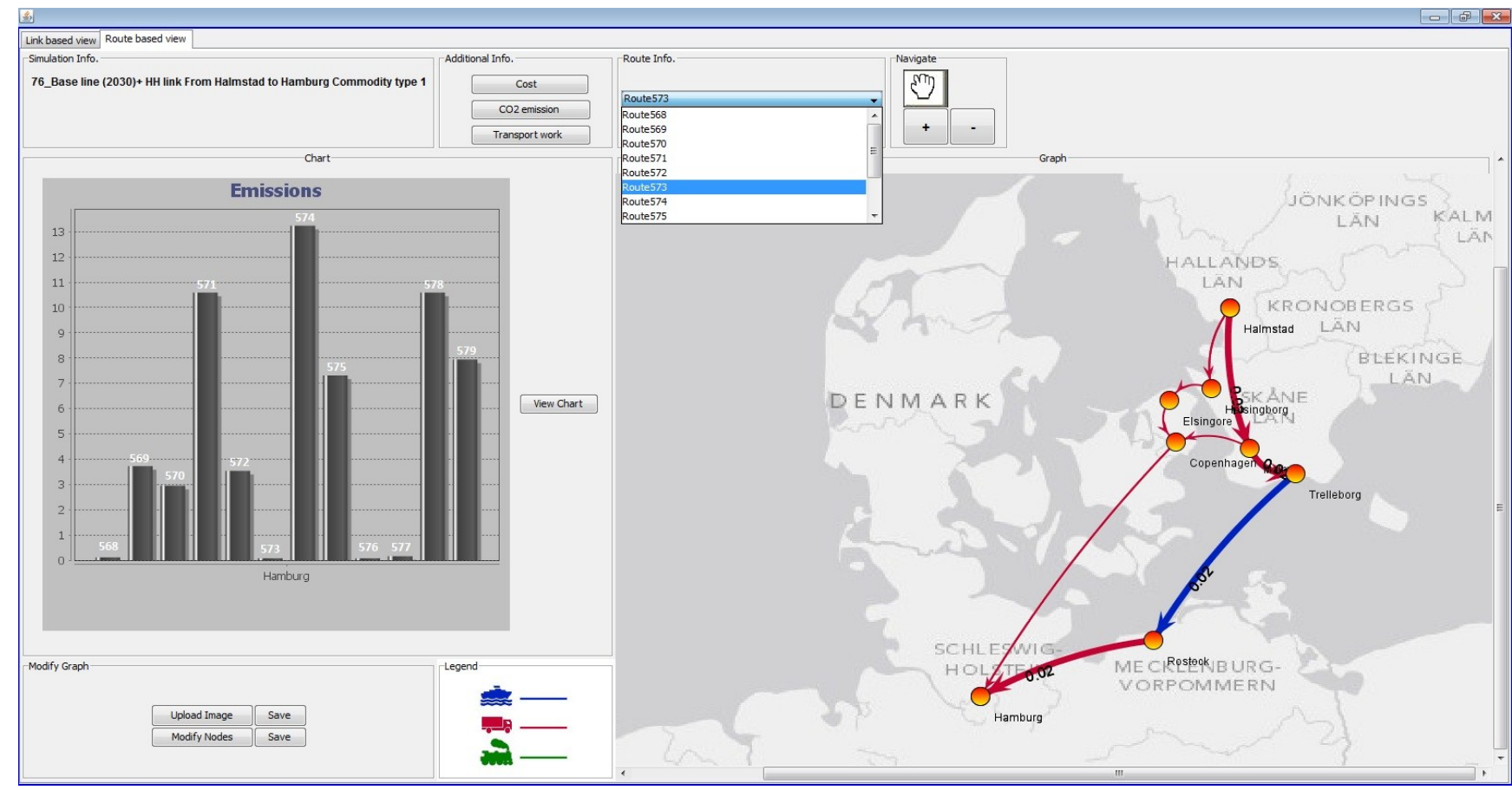

Figure 1: A screenshot of the visualization toolkit. (Showing route-based view; the selected route is highlighted by a thicker line)

could finalize valuable design decisions, such as the form of demonstrating different transportation modes and using graph to display the transportation stakeholders as nodes of the transportation network. We propose to visualize information of each main output (Cost, $\mathrm{CO}_{2}$, Transport work) and allow users to select the desired information as it is shown on the top of the Figure 1. In addition, different edge colors are used to exemplify the various transport modes of the links. The bar chart in the left side of the Figure 1 compares the amount of $\mathrm{CO}_{2}$ emissions generated on each route. In general, main functionalities of the system, regarding user requirements are designed as below:

- Func1. Show as a graph: Using color, edge thickness to differentiate links and routes and to show the data.

- Func2. Draw charts: to illustrate data and make comparison between different scenarios.

- Func3. Link and route based information on Graph: Showing information of cost, $\mathrm{CO}_{2}$ emission, amount of transport work on each link and route.

- Func4: Interactivity. The interactivity of the visualization toolkit empowers users to explore implications of different simulation scenarios and engages them in what-if- reasoning.

- Func5. Add a map as background: to increase usability and readability of the transportation data.

\section{CONCLUSION AND FUTURE WORK}

Based on the positive feedbacks received during presentation of the toolkit to users, we believe that the visualization toolkit contributes to improvement of the understanding and insight of the transportation analysis stakeholders and policy makers into transportation simulation data, and consequently empower them in the decision making process. Compared to the traditional methods for communicating the results of transportation simulation, the interactive visualization toolkit enhances the understandability of simulation results in a number of ways, e.g. the capability to compare the results of different simulation scenarios and to retrieve the information related to cost, the amount of transport work and $\mathrm{CO}_{2}$ emissions on each route and transport link.

A possible future improvement of the visualization toolkit can lie in visualizing the input of transportation simulation systems in order to design different scenarios graphically.

\section{REFERENCES}

1. Brunner, D., Cross, G., McGhee, C., Levis, J. and Whitney, D. Toward increased use of simulation in transportation. Winter Simulation Conference, 1998.

2. Chen, C. Information Visualization: Beyond the Horizon, 2nd edition, Springer, 2004.

3. Hoen, P.J., et al. Simulation and visualization of a market-based model for logistics management in transportation, Proc. AAMAS'04, pp. 1218-1219.

4. Holmgren, J., Davidsson, P., Ramstedt, L. and Persson, J.A. TAPAS: A Multi-Agent-Based Model for Simulation of Transport Chains. Simulation Modelling Practice and Theory, Elsevier, 2012.

5. Hughes, R.G. Toward an Expanded Research Agenda for Visualization in Transportation: Incorporating SAFETEA-LU Directives for 'Planning'. Institute for Transportation Research and Education, North Carolina State University, Raleigh, NC, 2008. 